\title{
Suture Tape Augmentation Has No Effect on Anterior Tibial Translation, Gap Formation, or Load to Failure of Anterior Cruciate Ligament Repair: A Biomechanical Pilot Study
}

\author{
Charles Qin, M.D., Adam Kahn, M.D., Farid Amirouche, Ph.D., Amir Beltagi, Ph.D., \\ Sonia Pradhan, B.S., Jason L. Koh, M.D., and Aravind Athiviraham, M.D.
}

\begin{abstract}
Purpose: The purpose of our pilot study was to assess the effect of augmenting anterior cruciate ligament (ACL) repair with suture tape on biomechanical parameters including anterior tibial translation, gap formation, and load to failure. Methods: Ten fresh-frozen nonpaired cadaveric knees were dissected, and baseline anterior-posterior stability of both ACL-intact and -deficient knees was obtained. The specimens were randomized to undergo ACL repair either with or without suture tape reinforcement, and anterior tibial translation, as well as gap formation, was measured after cyclic loading. Finally, all specimens were subjected to a single pullout force to determine maximum load to failure. We performed $t$ test analysis to compare means between groups, and significance was defined as $P<.05$. Results: On $t$ test analysis, no statistically significant difference was found regarding anterior tibial translation between the ACL-intact group and either repair group or between the repair group without suture tape augmentation and the repair group with suture tape augmentation. No significant difference in gap formation was detected between the repair groups with and without suture tape augmentation at 100 cycles $(1.25 \mathrm{~mm}$ vs $1.02 \mathrm{~mm}, P=.6), 250$ cycles $(2.87 \mathrm{~mm}$ vs $2.12 \mathrm{~mm}, P=.3)$, and 500 cycles $(4.5 \mathrm{~mm}$ vs $4.55 \mathrm{~mm}, P=.5)$. The average load to failure of the repairs without suture tape augmentation was not significantly different from that of the repairs with suture tape augmentation $(725.9 \mathrm{~N}$ vs $725.7 \mathrm{~N}, P=.99)$. Conclusions: In this pilot study, we did not identify a difference between ACL repairs with and without suture tape augmentation regarding anterior tibial translation, gap formation, or maximum load to failure. Clinical Relevance: Treatment of ACL tears with primary ACL repair is a highly debated topic, and studies such as this study to further our understanding of the biomechanical properties of augmented ACL repairs are important for surgeons when deciding the best treatments for their patients.
\end{abstract}

From the Department of Orthopaedic Surgery and Rehabilitation, University of Chicago, Chicago, Illinois, U.S.A. (C.Q., A.K., A.A.); Department of Orthopaedic Surgery, University of Illinois at Chicago, Chicago, Illinois, U.S.A. (F.A., A.B., S.P.); and Orthopaedic and Spine Institute, NorthShore University Health System, Chicago, Illinois, U.S.A. (J.L.K.).

The authors report the following potential conflicts of interest or sources of funding: A.A. has served as a laboratory instructor for an Arthrex-sponsored anterior cruciate ligament repair course and previously received education, travel, lodging, and food/beverage support from Arthrex, outside the submitted work. Full ICMJE author disclosure forms are available for this article online, as supplementary material.

Received April 8, 2020; accepted September 19, 2020.

Address correspondence to Charles Qin, M.D., Department of Orthopaedic Surgery and Rehabilitation, University of Chicago, 5841 S Maryland Ave, Chicago, IL 60637, U.S.A.E-mail: Charlesq2493@gmail.com

Published by Elsevier Inc. on behalf of the Arthroscopy Association of North America. This is an open access article under the CC BY-NC-ND license (http://creativecommons.org/licenses/by-nc-nd/4.0/).

2666-061X/20477

https://doi.org/10.1016/j.asmr.2020.09.016
A lthough short-term clinical results after primary repair of anterior cruciate ligament (ACL) tears reported in the historic literature were promising, longer-term follow-up of these studies showed inferior results compared with reconstruction. ${ }^{1}$ Since, attempts to improve the results of repair have focused attention on case selection and single-bundle augmentation. In fact, positive clinical outcomes after primary repair of proximal ACL tears have been reported in the recent literature. $^{2-4}$

The advantages of primary repair include preservation of proprioception, normal kinematics, and availability of ACL reconstruction as a salvage procedure..$^{5-7}$ The variety of repair methods and adjunctive techniques available has sparked a contemporary resurgence into preclinical investigation of ACL repair. ${ }^{7-12}$ Gap formation of $1 \mathrm{~mm}$ after repetitive cycling was reported after ACL repair with suture anchor and 
button fixation techniques. ${ }^{7}$ Recent attention has been paid to internal suture tape augmentation of ACL repairs in an attempt to improve the mechanical properties of the native ACL without stress shielding it. ${ }^{13,14}$ Loads to failure greater than $600 \mathrm{~N}$ have been reported for ACL repairs with static internal suture tape augmentation in porcine models. ${ }^{15}$

A better biomechanical understanding of postoperative primary ACL repairs in cadaveric models will help to guide further clinical research on optimal treatment techniques. The purpose of our pilot study was to assess the effect of augmenting ACL repair with suture tape on biomechanical parameters including anterior tibial translation, gap formation, and load to failure. Our hypothesis was that augmenting ACL repair with suture tape would lead to decreased anterior tibial translation, decreased gap formation, and increased load to failure compared with ACL repair without augmentation.

\section{Methods}

This study did not require review and/or approval from our institution's review board.

\section{Preliminary Study}

A preliminary analysis of 6 separate fresh porcine knee specimens was performed to determine the preferred suture configuration to be used in our study, given the varying described techniques of suture preparation of the ACL in the literature. ${ }^{11,12}$ We recognize that the most common mode of failure of ACL repairs in biomechanical models is not suture slipping at the suture-tissue interface but rather suture slipping in the femoral tunnel. ${ }^{7}$ The ACL femoral footprint was transected in all 6 porcine knee specimens, and 2 specimens each were prepared with a "luggage-tag" stitch, Bunnell stitch, and Krackow stitch with No. 2 FiberWire (Arthrex, Naples, FL), in a manner that could be replicated arthroscopically. The specimen was then mounted onto an MTS machine (MTS Systems, Eden Prairie, MN) and subjected to a load rate of $1 \mathrm{~mm} / \mathrm{s}$ perpendicular to the specimen, which purely applied a distraction force to determine load to failure. A photograph of the experimental setup can be found in Figure 1. The mean yield point was greatest with the Krackow suture configuration, at $165 \pm 21.1 \mathrm{~N}$, compared with $77.8 \pm 14.5 \mathrm{~N}$ for the luggage-tag stitch and 101.4 $\pm 17.8 \mathrm{~N}$ for the Bunnell stitch, and all failures occurred at the suture-tissue interface. On the basis of these results, we used a Krackow stitch as the preferred method to capture remnant ACL tissue.

\section{Cadaveric Preparation}

Ten fresh-frozen nonpaired cadaveric knees were used in this biomechanical study. All specimens were systematically assessed for damage by the senior author
(A.A.) and were excluded if there was damage to the ACL. Ultimately, the integrity of the ACL was preserved in all specimens, and no specimens required exclusion. The surrounding soft tissues, including the patella and extensor mechanism, were dissected off the specimens, leaving the femur, tibia, and cruciate ligaments intact. $^{14,16}$ During dissection, care was taken to not disturb the femoral and tibial footprints of both bundles of the ACL.

To obtain baseline anterior-posterior stability of both ACL-intact and ACL-deficient knees, all native cadaveric knees were mounted onto the MTS machine (Fig 2). A compressive force of $50 \mathrm{~N}$ was applied at $30^{\circ}$ of knee flexion, and an anteriorly directed average force of $137 \mathrm{~N}$ was applied by a dynamometer through a pulley and hook placed at the center of the tibial tubercle. ${ }^{17}$ Similar to the methodology of de Carvalho et al., ${ }^{17}$ the OptiTrack motion capture system (NaturalPoint, Corvallis, OR) was used to define unit vectors created by 2 defined points of the femur (posteriormost aspect of the lateral femoral condyle and posterior-most aspect of the medial femoral condyle) and tibia (posterior-most aspect of the lateral tibial plateau and posterior-most aspect of the medial tibial plateau), and the sagittal distance between the midpoints of these 2 vectors was calculated. However, unlike de Carvalho et al., we did not assess the individual contribution of each bundle to the ACL on anterior tibial translation. A No. 15 blade was used to sharply

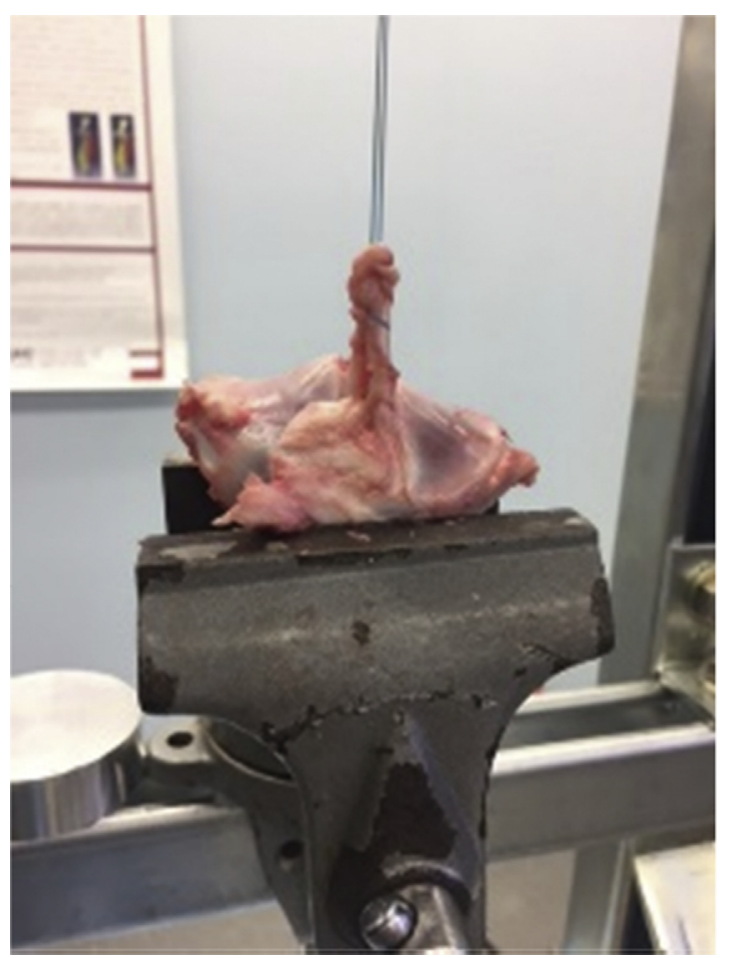

Fig 1. Test setup of porcine tibia with simulated anterior cruciate ligament tear captured with Krackow locking suture. 


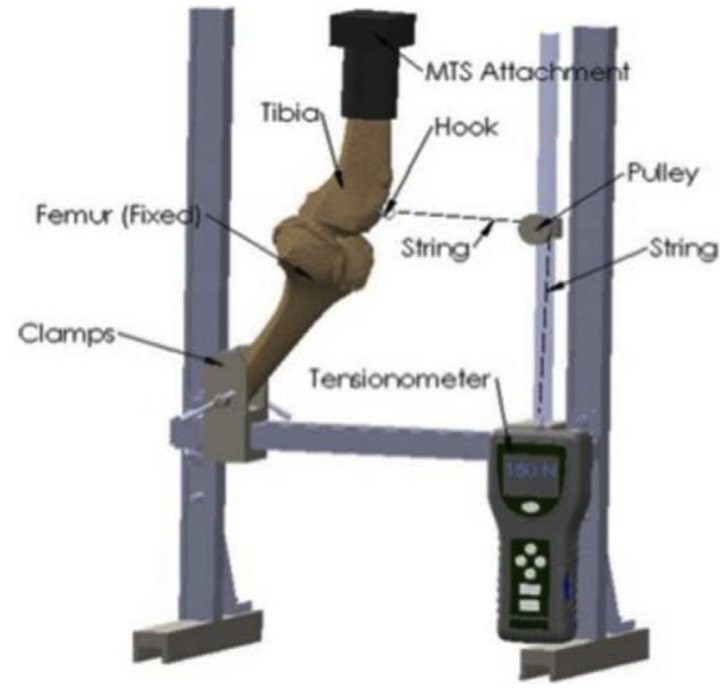

Fig 2. Test setup of cadaveric knee after anterior cruciate ligament repair with femur clamped and tibia secured in cylindrical fixture.

transect the ACL of each specimen at its femoral footprint, and anterior tibial translation was measured again using the aforementioned methodology.

\section{Surgical Technique}

All 10 cadaveric knee specimens underwent simulated ACL rupture. We introduced a No. 15 blade to sharply transect the ACL at its femoral footprint. Five specimens were randomized to undergo repair without suture tape reinforcement. The ACL remnant was prepared with a Krackow stitch. With the knee at $90^{\circ}$ of flexion, a femoral tunnel was drilled at the femoral origin of the ACL, exiting the lateral femoral cortex, and the 2 limbs of the Krackow stitch were pulled through the tunnel. A 4.75-mm hole was tapped and punched at the lateral metaphyseal flare, and a corresponding SwiveLock anchor (Arthrex) was introduced and deployed while the sutures were tensioned at $30^{\circ}$ of knee flexion with a posterior drawer force. ${ }^{11}$ Photographs of the repaired specimen can be found in Figure 3.

Five specimens underwent repair with suture tape reinforcement using the InternalBrace concept (Arthrex). The technique was similar to the technique described earlier but with the addition of independently tensioned FiberTape (Arthrex) to SwiveLock suture anchor fixation on the tibial side and Tight Rope RT fixation (Arthrex) on the femoral side. ${ }^{14,18}$ The FiberTape was independently tensioned with maximal manual load to reproduce a clinically relevant setting with the knee in full extension; this was done prior to tensioning the repair. Photographs of the repaired specimen with suture tape augmentation can be found in Figure 4.

\section{Cadaveric Testing}

All prepared specimens were mounted onto the MTS machine. The knees were not preconditioned. They underwent 100, 250, and 500 cycles from $90^{\circ}$ of flexion through full extension with a frequency of $0.1 \mathrm{~Hz}$, after which gap formation was determined with a digital caliper by measuring the distance between the femoral origin of the native ACL and the repaired ACL. This was performed by the senior collaborator (F.A.). The frequency of loading used in our study to determine gap formation at 100, 250, and 500 cycles was derived from a previous study evaluating primary ACL repair. ${ }^{7}$ Anterior tibial translation of all specimens was measured after cyclic loading using the protocol
Fig 3. Anteroposterior (A) and lateral (B) views of cadaveric anterior cruciate ligament repair without suture augmentation. Suture anchor fixation at the lateral metaphysis is shown.
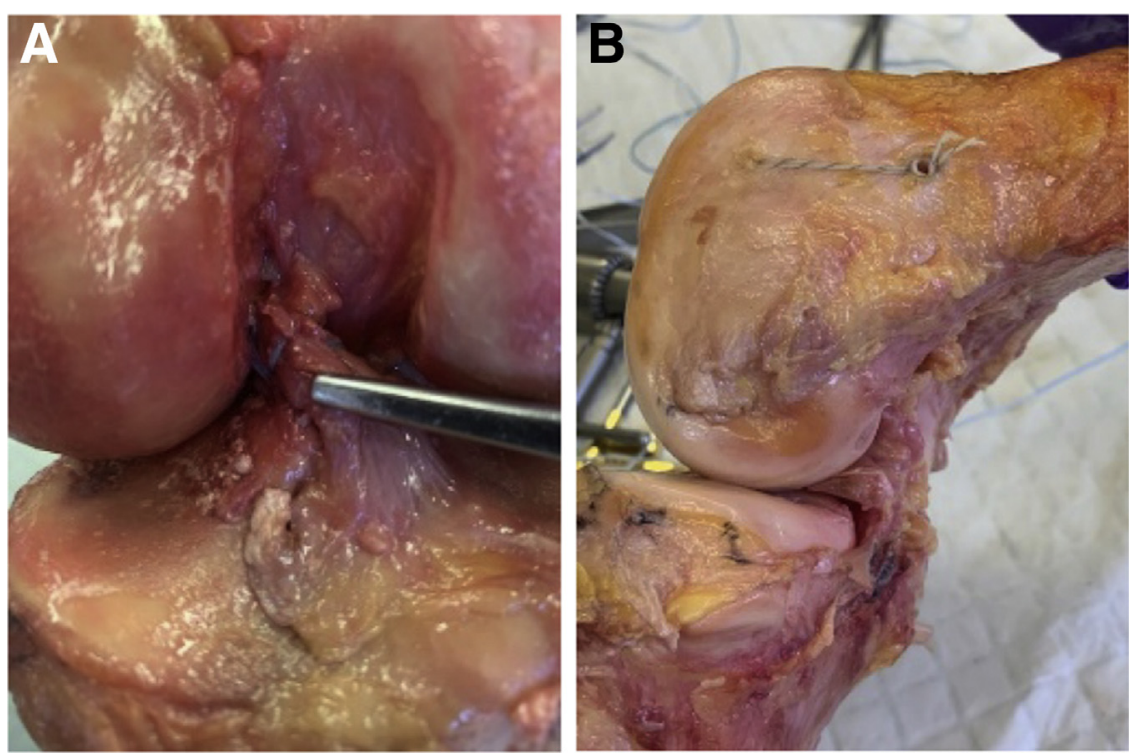

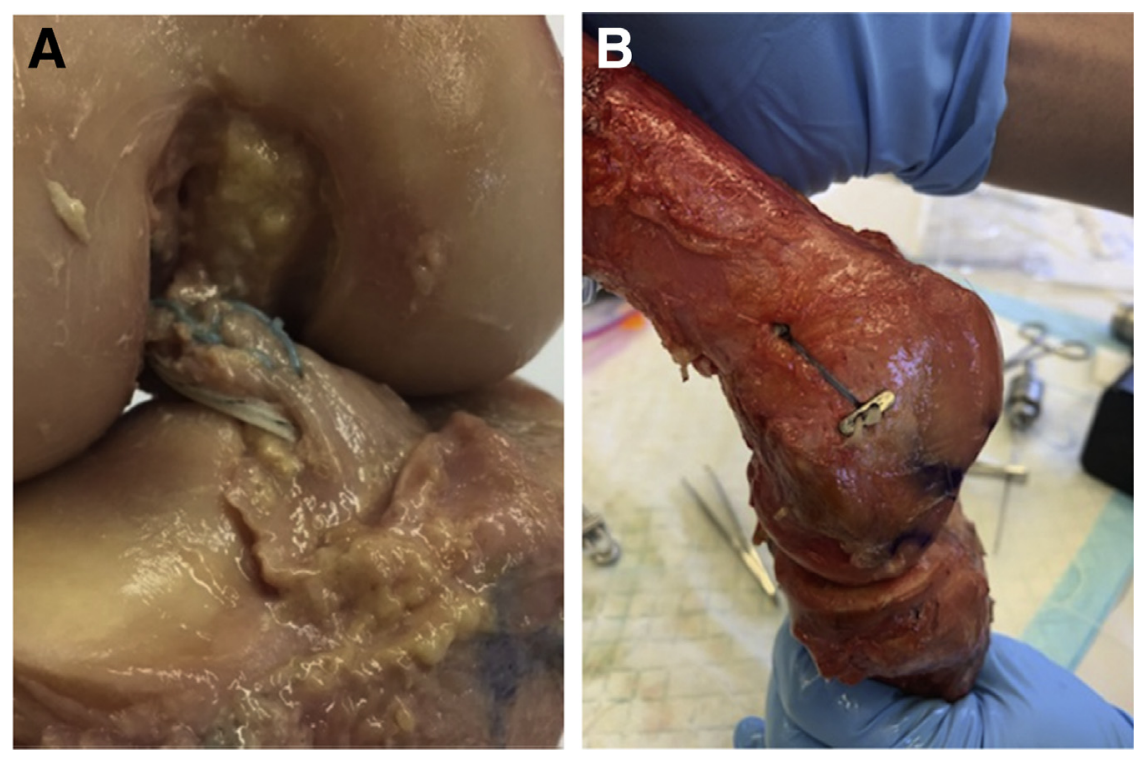

Fig 4. Anteroposterior (A) and lateral (B) views of cadaveric anterior cruciate ligament repair with suture augmentation. Button fixation at the lateral metaphysis is shown.

described earlier in the article. Finally, while in full extension, all specimens were subjected to a pullout test force at a load rate of $1 \mathrm{~mm} / \mathrm{s}$ perpendicular to the femur to determine the maximum load to failure. ${ }^{7}$ The failure mode was documented.

\section{Statistical Analysis}

An a priori power analysis was performed to determine the minimum sample size needed to detect a difference, assuming an effect size of 0.8 , power of 0.8 , and probability level of .05 . The minimum sample size needed was 56.

Anterior tibial translation, gap formation, and maximum load to failure were reported as mean and standard deviation (SD). Regarding the statistical analysis of anterior tibial translation, we aimed to compare each test state (ACL tear, ACL repair with augmentation, and ACL repair without augmentation) with the ACL-intact group. This generated 3 pair-wise comparisons, best analyzed with the Student $t$ test. Furthermore, $t$ test analysis was used to compare mean values of gap formation and maximum load to failure between groups. Statistical significance was defined as $P<.05$.

\section{Results}

\section{Specimens}

All 10 specimens were from male cadavers. The average age was 75.8 years (SD, 14 years), and the average weight was $158.4 \mathrm{lb}$ (SD, $26 \mathrm{lb}$ ). There were 4 left and 6 right knee specimens.

\section{Anterior Tibial Translation}

Data on anterior tibial translation are available in Table 1. Mean anterior tibial translation was $15.2 \pm 3.1$ $\mathrm{mm}$ in the ACL-intact specimens, $22.3 \pm 3.9 \mathrm{~mm}$ in the
ACL tear group, $16.4 \pm 2.5 \mathrm{~mm}$ in the repair group without suture tape augmentation, and $18.0 \pm 1.9 \mathrm{~mm}$ in the repair group with suture tape augmentation. On $t$ test analysis, no statistically significant difference was found between the ACL-intact group and either repair group $(P=.44$ and $P=.37)$ or between the repair group without suture tape augmentation and the repair group with suture tape augmentation $(P=.29)$. There was a statistically significant difference between the ACL tear group and the ACL-intact group $(P=.023)$.

\section{Gap Formation}

Gap formation data are available in Table 2. Mean gap formation in the repair group without suture tape augmentation was $1.25 \pm 0.8 \mathrm{~mm}$ at 100 cycles, $2.87 \pm$ $1.2 \mathrm{~mm}$ at 250 cycles, and $4.5 \pm 1.6 \mathrm{~mm}$ at 500 cycles. Mean gap formation in the repair group with suture tape augmentation was $1.02 \pm 0.3 \mathrm{~mm}$ at 100 cycles, $2.12 \pm$ $0.48 \mathrm{~mm}$ at 250 cycles, and $4.55 \pm 1.5 \mathrm{~mm}$ at 500 cycles. Via the Student $t$ test, no significant differences in gap formation were detected between the repair groups with and without suture tape augmentation.

\section{Load to Failure}

Load-to-failure values and the failure modes of the 10 repaired specimens are recorded in Table 3. The average load to failure of the repairs without suture tape augmentation was $725.9 \pm 353.9 \mathrm{~N}$, and the average load to failure of the repairs with suture tape augmentation was $725.7 \pm 219.9 \mathrm{~N}$. No difference in load to failure was found between the groups on $t$ test analysis $(P=.99)$.

\section{Discussion}

The main finding in our pilot study was that there was no difference in anterior tibial translation, gap formation, and ultimate load to failure between ACL repairs 
Table 1. Anterior Tibial Translation

\begin{tabular}{ccccc}
\hline & Intact ACL & ACL Tear & Repair Without Suture Augmentation & Repair With Suture Augmentation \\
\hline Specimen No. & & & & 18.5 \\
1 & 11.1 & 17.4 & 12.3 & NA \\
2 & 17.7 & 23.2 & 18.5 & NA \\
3 & 15.3 & 20.4 & 16.4 & NA \\
4 & 9.7 & 17.1 & 16.2 & NA \\
5 & 14.4 & 20.8 & NA & NA \\
6 & 14.9 & 25.4 & NA & 17.7 \\
7 & 16.4 & 21.2 & NA & 16.7 \\
8 & 19.8 & 29.0 & NA & 20.9 \\
9 & 14.7 & 21.8 & NA & 16.1 \\
10 & 18.0 & 26.9 & 16.4 & 18.5 \\
Average & 15.2 & 22.3 & 2.5 & 18.0 \\
SD & 3.1 & 3.9 & 1.9 \\
\hline
\end{tabular}

NOTE. On $t$ test analysis, comparison of the ACL-intact group versus specimens with ACL tears showed $P=.023$; ACL-intact group versus ACL repair group without suture augmentation, $P=.44$; ACL-intact group versus ACL repair group with suture augmentation, $P=.37$; and ACL repair groups without suture augmentation versus with suture augmentation, $P=.29$.

ACL, anterior cruciate ligament; NA, not applicable; SD, standard deviation.

with and without suture tape augmentation, which was contrary to our hypothesis. Biomechanical studies have shown promising results regarding ACL repair in both animal and human models. ${ }^{1,7,10,19,20}$ ACL repair has been shown to re-create the kinematics of the native knee and present superior anteroposterior stability compared with reconstruction in 1 recent study. ${ }^{6,21,22}$ Van der List and DiFelice ${ }^{7}$ reported that cadaveric ACL repairs with suture button and suture anchor fixation had a mean load to failure of $243 \mathrm{~N}$, which is well over the minimum of $60 \mathrm{~N}$ necessary for early range of motion. ${ }^{23}$ Bachmaier et al. ${ }^{15}$ showed ultimate load-tofailure values of over $800 \mathrm{~N}$ in porcine specimens with internally braced ACL repairs with button fixation and over $600 \mathrm{~N}$ in internally braced repairs with suture anchor fixation, which are more in line with the load to failure reported in our study. Differences in load to failure across studies may reflect the heterogeneity of constructs studied, including technique of fixation, ACL suturing, and augmentation. In addition, given that failure of almost all specimens in our study occurred as the suture pulled out its fixation through the femoral tunnel, our findings likely reflect the failure load of the knotless fixation technique more so than the suture tape augmentation. For example, a study on patellar tendon repair with suture anchor fixation with and without InternalBrace augmentation reported maximum loads similar to those of our study. ${ }^{24}$
Clinical outcomes after ACL repair are mixed. A recent meta-analysis authored by van der list et al. ${ }^{10}$ concluded that the current literature shows low complication rates and high functional scores after ACL repair but is limited by short follow-up and a high risk of selection and publication bias. More recently, studies have shown an increased risk of rerupture in adolescents and in subsequent surgery for mostly cyclops lesions in adults. ${ }^{1,20}$

Descriptions of suture configurations in preparing the ACL remnant in repairs are varied in the literature. ${ }^{11,25}$ Evidence to support one method over another remains anecdotal at this point. Comparison of the strength of locking sutures can be found in the flexor tendon literature, which has shown that the number of suture strands crossing the repair site is more important than the suture configuration itself. ${ }^{26,27}$ Furthermore, the type of suture configuration must be compatible within the limits to prepare this configuration arthroscopically using a suture passer. In the preliminary study we completed to optimize the selected ACL repair technique, we compared the mean yield points of 3 groups of porcine ACL specimens with different suture configurations that were compatible with arthroscopic placement and subsequently loaded to failure. The mean yield point was greatest with the Krackow suture configuration; all failures occurred at the suture-tissue interface. Further investigation into the optimal

Table 2. Gap Formation

\begin{tabular}{|c|c|c|c|}
\hline Cycles & Repair Without Suture Augmentation, mm & Repair With Suture Augmentation, mm & $P$ Value \\
\hline 100 & $1.25(0.8)$ & $1.02(0.3)$ & .6 \\
\hline 250 & $2.87(1.2)$ & $2.12(0.48)$ & .3 \\
\hline 500 & $4.5(1.6)$ & $4.55(1.5)$ & .5 \\
\hline
\end{tabular}

NOTE. Data are presented as mean (standard deviation). 
Table 3. Load to Failure

\begin{tabular}{lcl}
\hline & Load to Failure, $\mathrm{N}$ & Failure Mode \\
\hline Individual specimen data & & $P$ Value \\
Without suture augmentation & $1,157.7$ & Suture slipped through femoral tunnel \\
& 594.9 & Suture slipped through femoral tunnel \\
& 276 & Suture slipped through femoral tunnel \\
With suture augmentation & $1,005.1$ & Suture slipped through femoral tunnel \\
& 595.8 & Suture slipped through femoral tunnel \\
& 503.5 & Suture slipped through femoral tunnel \\
Comparison of repair groups & 897.5 & Suture slipped through femoral tunnel \\
Without suture augmentation, mean (SD) & 856.7 & Suture slipped through femoral tunnel \\
With suture augmentation, mean (SD) & 902.3 & Suture slipped through femoral tunnel \\
\hline SD, standard deviation. & 468.6 & \\
\end{tabular}

SD, standard deviation.

suturing technique of the ACL must take into account that the most common mode of ACL repair failure in previous biomechanical studies was suture slipping in the femoral tunnel rather than at the suture-tissue interface.

\section{Limitations}

This study is not without limitations. The lack of a demonstrable statistical difference in the $26 \%$ reduction in mean gap formation at 250 cycles with suture tape augmentation ( $2.87 \mathrm{~mm}$ vs $2.12 \mathrm{~mm}$ ) may have been due to a type II error. Furthermore, a $40 \%$ increase in maximum load to failure in standard graft-diameter ACL reconstructions with suture tape reinforcement has been shown previously, ${ }^{14}$ whereas our study did not detect a difference. The discrepancy may also be attributable to the fixation technique, given that we used a knotless suture anchor fixation in the lateral metaphysis. We acknowledge that there may be variation in the preferred femoral fixation of the ACL repair in the clinical setting, which may affect the generalizability of our results to other forms of fixation.

Regarding determining anterior tibial translation, the anterior force applied through a pulley and hook system in the tibial tubercle was standardized at $137 \mathrm{~N}$ for each specimen. However, the distance between the tibial tubercle and joint line for each specimen would likely vary according to expected variations in anatomy, which may lead to slight variations in the force vector.

Our model assesses time-zero anterior tibial translation, gap formation, and maximum load to failure of ACL repair specimens and cannot account for biological healing and neuromuscular adaptations. Moreover, suture tape augmentation may play a role in stabilization of the ACL-repaired knee in rotation, which was not studied here but is an avenue for future investigation. Previous literature has shown that maximum load to failure increases significantly from 2 weeks to 4 months postoperatively. ${ }^{28}$ Furthermore, data from sheep models have shown the biomechanical superiority of suture tape-augmented ACL repairs only, at 16 weeks and beyond, as the InternalBrace protected the repaired ligament during the healing phase. ${ }^{29}$

Finally, the external validity of our study results is weakened by the demographic characteristics of the cadaveric specimens used, as well as the use of ACL transection to model proximal ACL ruptures. Young, active patients with proximal tears with excellent tissue quality are generally selected for ACL repair, whereas the average age of specimens in this study was 76 years; moreover, all the specimens were from male cadavers and were not paired. Because the quality of the ACL tissue was assessed in each specimen and all failures during pullout testing occurred via suture pullout through the femoral anchor, we do not feel that tissue quality contributed substantially to the results.

\section{Conclusions}

In this pilot study, we did not identify a difference between ACL repairs with and without suture tape augmentation regarding anterior tibial translation, gap formation, or maximum load to failure.

\section{References}

1. Gagliardi AG, Carry PM, Parikh HB, Traver JL, Howell DR, Albright JC. ACL repair with suture ligament augmentation is associated with a high failure rate among adolescent patients. Am J Sports Med 2019;47:560-566.

2. DiFelice GS, van der List JP. Arthroscopic primary repair of proximal anterior cruciate ligament tears. Arthrosc Tech 2016;5:e1057-e1061.

3. DiFelice GS, Villegas C, Taylor S. Anterior cruciate ligament preservation: Early results of a novel arthroscopic technique for suture anchor primary anterior cruciate ligament repair. Arthroscopy 2015;31:2162-2171. 
4. Achtnich A, Herbst E, Forkel P, et al. Acute proximal anterior cruciate ligament tears: Outcomes after arthroscopic suture anchor repair versus anatomic single-bundle reconstruction. Arthroscopy 2016:32:2562-2569.

5. Kiapour AM, Murray MM. Basic science of anterior cruciate ligament injury and repair. Bone Joint Res 2014;3: 20-31.

6. Schliemann B, Lenschow S, Domnick C, et al. Knee joint kinematics after dynamic intraligamentary stabilization: Cadaveric study on a novel anterior cruciate ligament repair technique. Knee Surg Sports Traumatol Arthrosc 2017:25:1184-1190.

7. van der List JP, DiFelice GS. Gap formation following primary repair of the anterior cruciate ligament: A biomechanical evaluation. Knee 2017;24:243-249.

8. Taylor SA, Khair MM, Roberts TR, DiFelice GS. Primary repair of the anterior cruciate ligament: A systematic review. Arthroscopy 2015;31:2233-2247.

9. Mahapatra P, Horriat S, Anand BS. Anterior cruciate ligament repair-Past, present and future. J Exp Orthop 2018;5:20.

10. van der List JP, Vermeijden HD, Sierevelt IN, DiFelice GS, van Noort A, Kerkhoffs G. Arthroscopic primary repair of proximal anterior cruciate ligament tears seems safe but higher level of evidence is needed: A systematic review and meta-analysis of recent literature. Knee Surg Sports Traumatol Arthrosc 2020;28:1946-1957.

11. van der List JP, DiFelice GS. Arthroscopic primary anterior cruciate ligament repair with suture augmentation. Arthrosc Tech 2017;6:e1529-e1534.

12. Boutsiadis A, Karampalis C, Tzavelas A, Vraggalas V, Christodoulou P, Bisbinas I. Anterior cruciate ligament remnant-preserving reconstruction using a "lasso-loop" knot configuration. Arthrosc Tech 2015;4:e741-e746.

13. Mackay GM, Blyth MJ, Anthony I, Hopper GP, Ribbans WJ. A review of ligament augmentation with the InternalBrace: The surgical principle is described for the lateral ankle ligament and ACL repair in particular, and a comprehensive review of other surgical applications and techniques is presented. Surg Technol Int 2015;26:239-255.

14. Bachmaier S, Smith PA, Bley J, Wijdicks CA. Independent suture tape reinforcement of small and standard diameter grafts for anterior cruciate ligament reconstruction: A biomechanical full construct model. Arthroscopy 2018;34:490-499.

15. Bachmaier S, DiFelice GS, Sonnery-Cottet B, et al. Treatment of acute proximal anterior cruciate ligament tears-Part 2: The role of internal bracing on gap formation and stabilization of repair techniques. Orthop J Sports Med 2020;8:2325967119897423.

16. Christel PS, Akgun U, Yasar T, Karahan M, Demirel B. The contribution of each anterior cruciate ligament bundle to the Lachman test: A cadaver investigation. J Bone Joint Surg Br 2012;94:68-74.
17. de Carvalho RT, Franciozi CE, Itami Y, et al. Bicruciate lesion biomechanics, part 1-Diagnosis: Translations over $15 \mathrm{~mm}$ at 90 degrees of knee flexion are indicative of a complete tear. Knee Surg Sports Traumatol Arthrosc 2019;27: 2927-2935.

18. Heusdens CHW, Hopper GP, Dossche L, Roelant E, Mackay GM. Anterior cruciate ligament repair with independent suture tape reinforcement: A case series with 2-year follow-up. Knee Surg Sports Traumatol Arthrosc 2019;27:60-67.

19. Vavken P, Fleming BC, Mastrangelo AN, Machan JT, Murray MM. Biomechanical outcomes after bioenhanced anterior cruciate ligament repair and anterior cruciate ligament reconstruction are equal in a porcine model. Arthroscopy 2012;28:672-680.

20. Hoogeslag RAG, Brouwer RW, Boer BC, de Vries AJ. Huis In 't Veld R. Acute anterior cruciate ligament rupture: Repair or reconstruction? Two-year results of a randomized controlled clinical trial. Am J Sports Med 2019;47: 567-577.

21. Kohl S, Evangelopoulos DS, Ahmad SS, et al. A novel technique, dynamic intraligamentary stabilization creates optimal conditions for primary ACL healing: A preliminary biomechanical study. Knee 2014;21:477-480.

22. Chahla J, Nelson T, Dallo I, et al. Anterior cruciate ligament repair versus reconstruction: A kinematic analysis. Knee 2020;27:334-340.

23. Seitz H, Wielke B, Schlenz I, Pichl W, Vecsei V. Load sharing in augmented anterior cruciate ligament repair: A mathematical analysis based on in vitro measurements. Clin Biomech (Bristol, Avon) 1996;1 1:431-438.

24. Rothfeld A, Pawlak A, Liebler SAH, Morris M, Paci JM. Patellar tendon repair augmentation with a knotless suture anchor internal brace: A biomechanical cadaveric study. Am J Sports Med 2018;46:1 199-1204.

25. Olmos MI, Sonnery-Cottet B, Barth J. How to succeed in arthroscopic anterior cruciate ligament primary repair? Step-by-step technique. Arthrosc Tech 2019;8:e37-e46.

26. Chauhan A, Palmer BA, Merrell GA. Flexor tendon repairs: Techniques, eponyms, and evidence. J Hand Surg Am 2014;39:1846-1853.

27. Haimovici L, Papafragkou S, Lee W, Dagum A, Hurst LC. The impact of Fiberwire, Fiberloop, and locking suture configuration on flexor tendon repairs. Ann Plast Surg 2012;69:468-470.

28. Cabaud HE, Feagin JA, Rodkey WG. Acute anterior cruciate ligament injury and repair reinforced with a biodegradable intraarticular ligament. Experimental studies. Am J Sports Med 1982;10:259-265.

29. Seitz H, Pichl W, Matzi V, Nau T. Biomechanical evaluation of augmented and nonaugmented primary repair of the anterior cruciate ligament: An in vivo animal study. Int Orthop 2013;37:2305-2311. 